\title{
Data loss for PLC of nonlinear systems Iterative Learning Control Algorithm
}

\author{
Yinjun Zhang ${ }^{1}$ Yinhui $\mathrm{Li}^{1} \quad$ Jianhuan $\mathrm{Su}^{2}$ Zhengjie $\mathrm{Lu}^{2}$ \\ ${ }^{1}$ Aeronautics and Astronautics Engineering Institute, Air Force Engineering University, Xi' an 710038, \\ Shanxi Province, \\ ${ }^{2}$ School of physics and electrical engineering Hechi university Yizhou GuangXi China
}

\begin{abstract}
When we use power line as data carrier, due to the complexity of the PLC network environment, data packet loss frequently, so the paper deal with the iterative learning control for a class of nonlinear systems with measurement dropouts in the PLC, and studies the P-type iterative learning control algorithm convergence issues, the data packet loss is described as a stochastic Bernoulli process, on this basis we given convergence conditions for the P-type iterative learning control algorithm. The theoretically analysis is supported by the simulation of a numerical example; the convergence of ILC can be guaranteed when some output measurements are missing.
\end{abstract}

\section{Introduction}

ILC (Iterative learning control) is a Human-simulated intelligent control method, these cyclical and repetitive tasks can be achieved fully tracked through continuous learning within finite time interval. Since 1984, Japanese scholars Arimoto proposed the method, ILC has been a hot issue at home and abroad, and has achieved rich theoretical results and application results [2-6]. Robustness is an important research aspect for iterative learning control and is also applied to solve the real factory problem. Due to the theoretical study of iterative learning control algorithm has the stringent system conditions which is the repeatability but the real system is difficult to meet these conditions, so robust study of ILC focused on non-strict repeat the conditions, such as the non-repetitive initial conditions [7], non-repetitive desired trajectory [8], non-repetitive disturbances and noise [910], non-repetitive uncertain time-delay [11-13] and so on..

At present, we use net to control in real factory control system, such as DCS, FCS and remote control based on Internet. Net control is a closed-loop system, although net control system, compared with the traditional control system, is low cost, easy installation and maintenance, system flexibility, ease of troubleshooting etc., but net control brings data delay, data dropout and other problems[14-15]. However, it is less applied to the net network with ILC, currently a small number of studies have mainly focus on the linear systems. In the literature [16-17], the lifting method is used to examine data loss of linear discrete systems and the design of robust convergence and algorithm of iterative learning control (ILC). Given that most working systems are nonlinear and the lifting method of generations of learning is not suitable for nonlinear discrete systems, the same method cannot be applied to nonlinear systems. Thus, the present paper is aimed at adopting a new approach to explore the convergence problem of ILC of nonlinear systems in case of data loss in the power line communication.

\section{Problem descriptions}

Consider the PLC MIMO non liner discrete time system:

$\left\{\begin{array}{l}x_{k}(t+1)=f\left(x_{k}(t)\right)+B\left(x_{k}(t)\right) u_{k}(t) \\ y_{k}(t)=g\left(x_{k}(t)\right)+D\left(x_{k}(t)\right) u_{k}(t)\end{array}\right.$

Here, $x_{k}(t) \in \mathfrak{R}^{n}, u_{k}(t) \in \mathfrak{R}^{m}, y_{k}(t) \in \mathfrak{R}^{r}$ are state vector, control input and output vector respectively. For the MIMO system, we give below suppose 1 .

Suppose 1 non-liner Function

$$
\begin{aligned}
& f(\cdot): \mathfrak{R}^{n} \times[0, N] \rightarrow \mathfrak{R}^{n}, g(\cdot): \mathfrak{R}^{n} \times[0, N] \rightarrow \mathfrak{R}^{n}, \\
& B(\cdot): \mathfrak{R}^{n} \times[0, N] \rightarrow \mathfrak{R}^{n \times m}, D(\cdot): \mathfrak{R}^{n} \times[0, N] \rightarrow \mathfrak{R}^{r \times m}
\end{aligned}
$$

the state vector $\mathrm{x}$ meets Lipschitz conditions, that is for

all $t \in[0, N]$ and any of $\left(x_{1}(t), x_{2}(t)\right)$

represent bounded constant $k_{F}, k_{B}, k_{G}, k_{D}$ meet

$\left\|f\left(x_{1}(t)\right)-f\left(x_{2}(t)\right)\right\| \leq k_{F}\left\|x_{1}(t)-x_{2}(t)\right\|$

$\left\|B\left(x_{1}(t)\right)-B\left(x_{2}(t)\right)\right\| \leq k_{E}\left\|x_{1}(t)-x_{2}(t)\right\|$

$\left\|g\left(x_{1}(t)\right)-g\left(x_{2}(t)\right)\right\| \leq k_{G}\left\|x_{1}(t)-x_{2}(t)\right\|$

$\left\|D\left(x_{1}(t)\right)-D\left(x_{2}(t)\right)\right\| \leq k_{D}\left\|x_{1}(t)-x_{2}(t)\right\|$

where $\|\cdot\|$ is Euclidean norm.

Suppose 2 Initial condition of system satisfies 
$x_{k}(0)=x_{d}(0)$, where $x_{k}(0)$ is the initial value of the state variable with $\mathrm{k}$ times, ${ }^{x_{d}}(0)$ is the initial value of the desired state.

Suppose 3 For the desired tracking trajectory $y_{d}(t)$, there are desired input $u_{d}(t)$ and $x_{d}(t)$ that meet formula (2)

$\left\{\begin{array}{l}x_{d}(t+1)=f\left(x_{d}(t)\right)+B\left(x_{d}(t)\right) u_{d}(t) \\ y_{d}(t)=g\left(x_{d}(t)\right)+D\left(x_{d}(t)\right) u_{d}(t)\end{array}\right.$

(2)

For the MIMO non-liner system (1), consider P-type learning control algorithm:

$$
u_{k+1}(t)=u_{k}(t)+\gamma e_{k}(t)
$$

$\gamma$ is learning gain matrix, $e_{k}(t)=y_{d}(t)-y_{k}(t)$ is system tracking error.

We assume output data $y_{k}(t)$ that is lost and each component of the output vector has same characteristics of loss. So the P-type ILC can be described as

$u_{k+1}(t)=u_{k}(t)+\gamma \Theta e_{k}(t)$

Where $\Theta=\left[\begin{array}{ccc}\eta_{1}(t) & \cdots & 0 \\ \vdots & \ddots & \vdots \\ 0 & \cdots & \eta_{r}(t)\end{array}\right]_{r \times r}, \eta(t)_{\text {is Bernoulli }}$

sequence, namely $\eta(t) \in\{0,1\}$, but $\eta(t)=0$ or $=1$ is

random, if $\eta(t)=0$ indicates that the data is lost, $\eta(t)=1$ means that no data is lost. Due to the randomness of data

loss, we know $\eta(t)$ and $u_{k}(t), x_{k}(t), y_{k}(t)$ are

unconnected. Assumption

$P\{\eta(t)=1\}=E\{\eta(t)\}=\bar{\eta}, P\{\cdot\}_{\text {is the probability, }} E\{\}_{\text {is }}$ desired factor. So $\bar{\eta}$ is the success rate of data

transmission and satisfies $0 \leq \bar{\eta} \leq 1$. Matrix $\Theta_{\text {meet }}$

$P\left\{\eta_{i}(t)=1\right\}=E\left\{\eta_{i}(t)\right\}=\bar{\eta} i=1,2, \cdots r$.

Before we give the convergence theorem, Let us make the following marks firstly:

$\|\cdot\|_{\infty}$ is infinite norm, we define vector $\mathrm{x}$ as

$\|x\|_{\infty}=\max _{1 \leq i \leq n}\left|\sum_{j=n}^{n} x_{j}\right|_{\text {, where }} x_{j}$ denotes the $\mathrm{j}$-th element of the vector $z$. for the $n \times n_{\text {matrix } \mathrm{M} \text {, we }}$

define $\|M\|_{\infty}=\max _{1 \leq i \leq n}\left|\sum_{j=n}^{n} m_{i, j}\right|, m_{i, j}$ denotes the element of matrix.

\section{Convergence analyses}

According to the below theorem we give the main result in the paper.
Theorem 1 For MIMO nonlinear system(1) to meet the suppose 1-3, using the formula(3) of ILC, when the system exits the output data dropout, if you choose the learning gain $\Gamma$ for all $\mathrm{t}$ and $\mathrm{k}$ satisfy $\left\|I-\bar{\eta} \gamma D\left(x_{k}(t)\right)\right\|_{\infty}<1$,

for all $t \in[0, N]$, we can get $\lim _{k \rightarrow \infty} E\left\{y_{k}(t)\right\} \rightarrow y_{d}(t)$.

Proof. By the formula(1) and suppose3, we know

$$
\left\{\begin{array}{l}
y_{d}(t)=c\left(x_{d}(t)\right)+d\left(x_{d}(t)\right) u_{d}(t) \\
y_{k}(t)=c\left(x_{k}(t)\right)+d\left(x_{k}(t)\right) u_{k}(t)
\end{array}\right.
$$

Eq.(4) minus Eq.(5), we get $e_{k}(t)$.

$$
\begin{aligned}
e_{k}(t)= & y_{d}(t)-y_{k}(t)= \\
& \left(g\left(x_{d}(t)\right)+D\left(x_{d}(t)\right) u_{d}(t)-\right. \\
& \left(g\left(x_{k}(t)\right)+D\left(x_{k}(t)\right) u_{k}(t)=\right. \\
& g\left(x_{d}(t)\right)-g\left(x_{k}(t)\right)+D\left(x_{d}(t)\right) u_{d}(t)- \\
& D\left(x_{k}(t)\right) u_{k}(t)= \\
& g\left(x_{d}(t)\right)-g\left(x_{k}(t)\right)+D\left(x_{k}(t)\right) d u_{k}(t) \\
& +\left[D\left(x_{d}(t)\right)-D\left(x_{k}(t)\right)\right] u_{d}(t)
\end{aligned}
$$

where $\delta u_{k}(t)=u_{d}(t)-u_{k}(t)$ according to the algorithm 3 , we get following Eq.

$\delta u_{k+1}(t)=u_{d}(t)-u_{k+1}(t)=$

$u_{d}(t)-u_{k}(t)-\gamma \eta(t) e_{k}(t)$,

Namely $\delta u_{k+1}(t)=\delta u_{k}(t)-\gamma \eta(t) e_{k}(t)=$

$$
\begin{aligned}
& =\left[1-\gamma \eta(t) d\left(x_{k}(t)\right)\right] \delta u_{k}(t) \\
& -\gamma \eta(t)\left[c\left(x_{d}(t)\right)-c\left(x_{k}(t)\right)\right]- \\
& \gamma \eta(t)\left[d\left(x_{d}(t)\right)-d\left(x_{k}(t)\right)\right] u_{d}(t)
\end{aligned}
$$

According to the suppose 1 and meet Lipschitz, we can get the below Eq. by Eq.(6).

$$
\begin{aligned}
& \left|\delta u_{k+1}(t)\right| \leq\left|1-\gamma \eta(t) d\left(x_{k}(t)\right)\right|\left|\delta u_{k}(t)\right|+ \\
& k_{c}|\gamma \eta(t)|\left|x_{d}(t)-x_{k}(t)\right| \cdot \\
& k_{d}|\gamma \eta(t)|\left|x_{d}(t)-x_{k}(t)\right|\left|u_{d}(t)\right|= \\
& \quad\left|1-\gamma \eta(t) d\left(x_{d}(t)\right)\right|\left|\delta u_{k}(t)\right|+k_{1}\left|\delta x_{k}(t)\right|
\end{aligned}
$$

Where $\delta x_{k}(t)=x_{d}(t)-x_{k}(t)$,

$$
k_{1}=k_{c}\left|\gamma \eta(t) \| k_{d} \gamma \eta(t)\right|\left|u_{d}(t)\right| \text {. }
$$

According to the Eq.(1)and suppose 3, we get

$$
\begin{aligned}
& \delta x_{k}(t)=x_{d}(t)-x_{k}(t)= \\
& f\left(x_{d}(t-1)\right)+b\left(x_{d}(t-1)\right) u_{d}(t-1)- \\
& f\left(x_{k}(t-1)\right)+b\left(x_{k}(t-1)\right) u_{k}(t-1),
\end{aligned}
$$

Then we get inequality (9) 


$$
\left|\delta x_{k}(t)\right| \leq
$$

$$
\begin{aligned}
& \left(k_{f}+k_{b}\left|u_{d}(t-1)\right|\right)\left|\delta x_{k}(t-1)\right|+ \\
& \left|b\left(x_{k}(t-1)\right)\right|\left|\delta u_{k}(t-1)\right|
\end{aligned}
$$

namely $\quad\left|\delta u_{k}(t) \leqslant k_{2}\right| \delta u_{k}(t-1)\left|+k_{3}\right| \delta u_{k}(t-1) \mid$

(9)

Where $k_{2}=\left|b\left(x_{k}(t-1)\right),, k_{3}=k_{f}+k_{b}\right| u_{d}(t-1) \mid$.

As $\left|\delta u_{k}(t)\right|=0$,so we expand right-hand member of Eq.(9) successively.

$$
\left|\delta u_{k}(t)\right| \leq \sum_{j=1}^{t} k_{2} k_{3}^{t-j}\left|\delta u_{k}(j-1)\right|
$$

Substitution Eq.(10) into Eq.(7),we get below Eq.(11).

$$
\begin{aligned}
& \left|\delta u_{k+1}(t)\right| \leq\left|1-\gamma \eta(t) d\left(x_{k}(t)\right)\right|\left|\delta u_{k}(t)\right|+ \\
& k_{1}\left|\delta x_{k}(t)\right| \leq\left|1-\gamma \eta(t) d\left(x_{k}(t)\right)\right|\left|\delta u_{k}(t)\right|+ \\
& k_{1} \sum_{j=1}^{t} k_{2} k_{3}^{t-j}\left|\delta u_{k}(j-1)\right|= \\
& \left|1-\gamma \eta(t) d\left(x_{k}(t)\right)\right|\left|\delta u_{k}(t)\right|+\sum_{i=0}^{t-1} K_{1, i}\left|\delta u_{k}(i)\right|
\end{aligned}
$$

here $K_{1, i}=K_{1} K_{2} K_{3}^{t-i-1}$, because Eq.(11) includes random factors $\eta(t)$, considering the $\eta(t)$ and $u_{k}(t)$ are unconnected. The two ends of the Eq.(11) take mathematic expectation, then we get Eq.(12)

$$
\begin{gathered}
E\left\{\left|\delta u_{k+1}(t)\right|\right\} \leq\left|1-\gamma \bar{\eta} d\left(x_{k}(t)\right)\right| E\left\{\left|\delta u_{k}(t)\right|\right\}+ \\
\sum_{i=0}^{t-1} \bar{K}_{1, i} E\left\{\left|\delta u_{k}(i)\right|\right\}
\end{gathered}
$$$$
\text { where } \bar{K}_{1, i}=E\left\{K_{1, i}\right\}=
$$$$
E\left\{\sum_{i=0}^{t-1} k_{c}|\gamma \eta(t)|\left(1+k_{d}\left|u_{d}(t)\right|\right) k_{2} k_{3}^{t-i-1}\right\}
$$$$
\sum_{i=0}^{t-1} k_{c}\left(|\gamma \bar{\eta}|+k_{d}\left|\gamma \bar{\eta} \| u_{d}(t)\right|\right) k_{2} k_{3}^{t-i-1}
$$

We take Eq.(12) expand from $\mathrm{t}=10$ to $\mathrm{t}=\mathrm{N}$ successively, then we get Eq.(13)

$$
\begin{aligned}
E\left\{\left|\delta u_{k+1}(0)\right|\right\} \leq & \left|1-\gamma \bar{\eta} d\left(x_{k}(0)\right)\right| E\left\{\left|\delta u_{k}(0)\right|\right\}, \\
E\left\{\left|\delta u_{k+1}(1)\right|\right\} \leq & \left|1-\gamma \bar{\eta} d\left(x_{k}(1)\right)\right| E\left\{\left|\delta u_{k}(1)\right|\right\}+ \\
& \sum_{i=1}^{N-1} \bar{K}_{1, i} E\left\{\left|\delta u_{k}(i)\right|\right\}
\end{aligned}
$$

$$
\begin{aligned}
& y_{k}(t)=0.2 \sin \left(x_{1, k}(t)\right)+0.1 x_{2, k}(t) \\
& \text { In the paper, we suppose that mathe } \\
& \text { output of the system is Eq. }(15) \\
& \overline{\bar{y}}_{d}(t)=\sin (\pi t / 100), t \in[0,500]
\end{aligned}
$$

Initial conditions set $X_{1, k}(t)=x_{2, k}(t)=0, u_{0}(k)=0$ in the simulation system. In both cases output data lose in the system. The first case is $\bar{\eta}=0.8,20 \%$ output data dropout, the second case is $\bar{\eta}=0.6,40 \%$ output data dropout. We choose P-type ILC: $u_{k+1}(t)=u_{k}(t)+0.5 e_{k}(t)$

In the non-liner system (14), $d\left(x_{k}(t)\right)=1$,we looked at convergence condition of theorem 1 in two data lose cases, when

We rewrite inequality(13) then get $V_{k+1} \leq A_{k} V_{k}$ $\bar{\eta}=0.8, \quad\left|1-\gamma \bar{\eta} d\left(x_{k}(k)\right)\right|=0.6<1$, algorithm convergence.

Simulation results are figure 1 and figure 2. Fig. 1 is maximum tracking error with different iteration time, obviously, when there is $20 \%$ measure output data lose, system tracing error still converges to 0. Fig.2 gives 
system output with different iteration. When $\bar{\eta}=0.6$

$$
\left|1-\gamma \bar{\eta} d\left(x_{k}(k)\right)\right|=0.7<1 \quad, \quad \text { algorithm }
$$

convergence remains the same, show fig. 3 and fig. 4

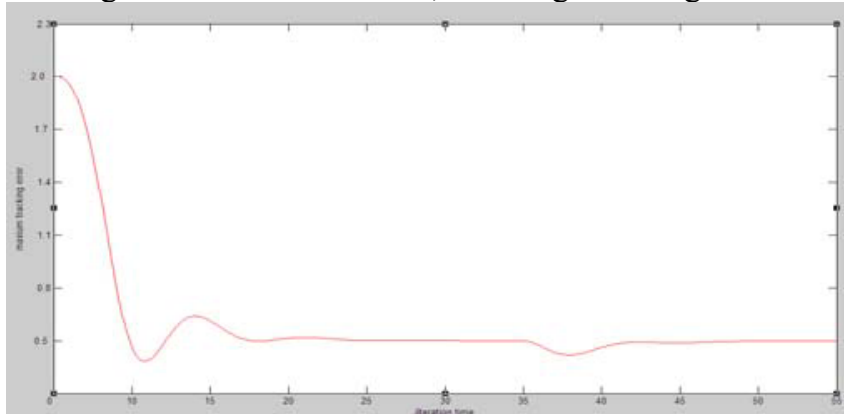

Fig. 1 the maximum tracking error with $20 \%$ data lose

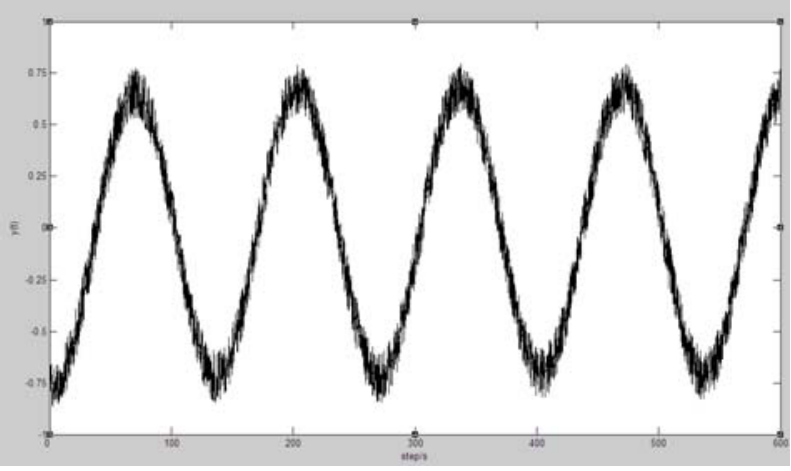

a) The fifth times iterations output

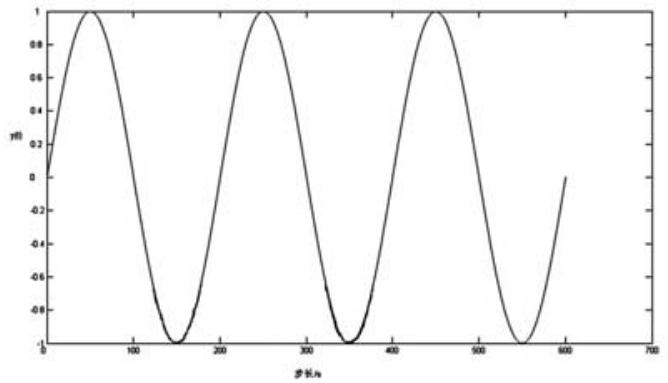

b) The twenty-fifth times iterations output

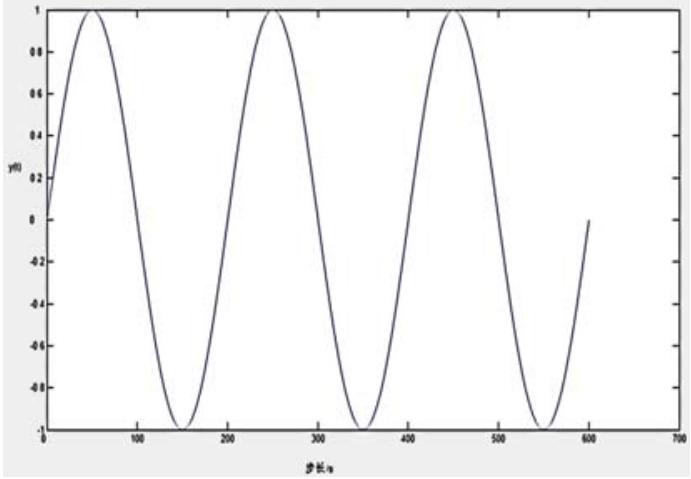

c) The thirty-fifth times iteration output

Fig. 2 The system output profiles of different iterations for $20 \%$ lose

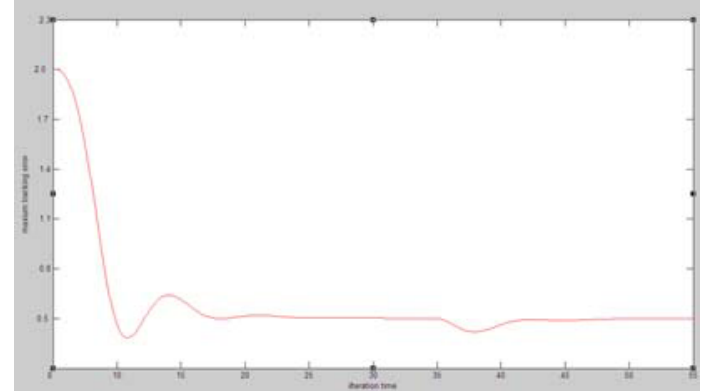

Fig. 3 the maximum tracking error with $40 \%$ data lose

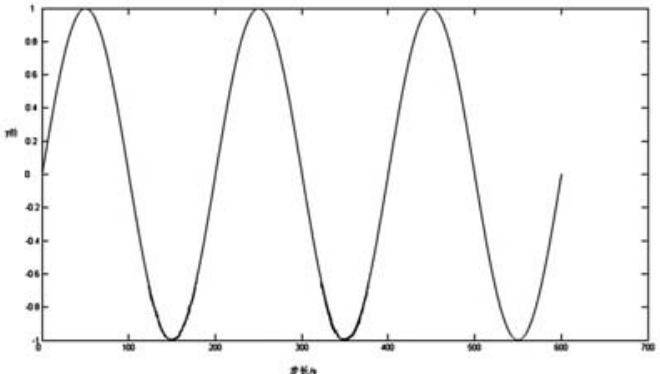

a) The thirty-fifth times iteration output

Fig.4 The system output profiles of different iterations for $40 \%$ lose

\section{Conclusions}

We research the presence of nonlinear system iteration learning control algorithm output measurement data loss robustness convergence problems in the paper and give the convergence condition. Theoretically proved that the convergence of the algorithm and the theoretical results through simulation. The results show that presence of certain nonlinear system when output data is lost, iterative learning control algorithm can guarantee the convergence of the tracking error, but the convergence rate increases will slow down with the degree of data loss.

\section{References}

1. ARIMOTO S, KAWAMURA S,MIYAZAKI F. Bettering operation of robotics [1]. Journal of Robotic System, 1984, 1 (2): 123 - 140.

2. XU J X. Analysis of iterative learning control for a class of nonlinear discrete-time system [J]. Automatic, 1997, 33(1 0): 1905 -1907

3. XU J X. Linear and Nonlinear Iterative Learning Control [M] Berlin: Springer Verlag,

4. 2003

5. AHN H S, CHEN Y Q, MOORE K L. Iterative learning control: brief survey and categorization [J]. IEEE Transactions on Systems, Man, and Cybernetics, Part C: Applicatiolls and Reviews, 2007, 37(6) 1099 -1121.

CHI R H, HOU Z S, XU J X. Adaptive ILC for a class of discrete time systems with iteration-varying trajectory and random initial condition[J]. Automatic, 2008, 44(8): 2207 - 2213

6. CHI Ronghu, HOU Zhongsheng, SUI Shulin. Nonparameter adaptive iterative learning control for the freeway traffic ramp metering[J]. Control Theory \& Applications, 2008, 25(6): 1011 - 1015. 
7. SUN M X, WANG D W. Initial shift issues on discrete-time iterative learning control with system relative degree [J]. IEEE Transactions on Automatic Control, 2003, 48(1): 144-148

8. YIN C K, XU J X, HOU Z S. A high-order internal model based iterative learning control scheme for nonlinear systems with time iteration-varying parameters [J]. IEEE Transactions on Automatic Control, 2010, 55(11)2665 - 2670

9. NORRLOF M, GUNNARSSON S. Disturbance aspects of iterative learning control $[\mathrm{J}]$. Engineering Applicatiol1s of Artificial Intelligellce, 2001 , 14(1): $87-94$.

10. SAAB SS. On a discrete-time stochastic learning control algorithm [J]. IEEE Transactions on Automatic Control, 2001, 46(8): 1333-1336.

11. MENG D, JIA Y, DU J, et al. Feedback approach to design fast iterative learning controller for a class of time-delay systems [J]. IET Control Theory and Applications, 2009, 3(2): 225 - 238.

12. CHEN W S, ZHANG L. Adaptive iterative learning control for nonlinearly parameterized systems with unknown time-varying delays $[\mathrm{J}]$. Intemational Joumal of Control, Automation, and Systems, 2010, 8(2): 177 - 186

13. CHEN Weisheng, WANG Yuanliang, LI Junmin. Adaptive learning control for nonlinearly parameterized systems with periodically timevarying delay [J]. Acta Automatica Sinica, 2008, 34(1 2): 1556- 1560.)

14. YANG T C. Networked control system: a brief survey [J]. IEE Proceedings Control Theory and Applications, 2006, 153(4): 403-412

15. Li Hongbo, SUN Zengqi, SUN Fuchun. Networked control systems: an overview of state-of-the-art and the prospect in future research $[\mathrm{J}]$. Control Theory \& Applications, 2010, 27(2): 238 - 243.

16. AHN H S, CHEN Y Q, MOORE K L. Discrete time intermittent iterative learning control with independent data dropouts [C] //Proceedings of the 17th 1FAC World Congress. Korea: IFAC, 2008: 12442-12447.

17. BU X H, HOU Z S. Stability of iterative learning control with data Dropout via asynchronous dynamical system[J].International Journal of Automation and Computing, 2011 ,8(1): 29 - 36.

Supported by KY2015LX339, 2015ER04, XJ2015QN001 